\title{
Interactive Design Exploration of Mobile Coal Logistics Management Platform*
}

\author{
Yan Shen \\ School of Arts \\ Northwest University \\ Xi'an, China 710000
}

\begin{abstract}
In the design of coal logistics platform, high quality user experience, good interactive operation habits and visual experience in line with user aesthetics have become the key factors of product success or failure. First of all, it is necessary to have a comprehensive understanding of user characteristics and focus on user experience. On this basis, the operation principle of interaction design shall be put forward to increase user's viscosity. Finally, based on satisfying the first two items and according to the user's operating habits as well as aesthetic habits, a set of high-fidelity drawings should be designed to meet the user's needs. By means of these principles, it is possible to make the design of coal logistics platform more standardized and normalized.
\end{abstract}

Keywords-coal freight; logistics management platform; interaction design; user experience

\section{INTRODUCTION}

No product is perfect, so, making best use of the advantages and bypassing the disadvantages helps users have a good experience. Even though there is no luxury interface, the simple and practical style should be kept. It is the core function of the product that retains customers. Accordingly, beautifying the content on the basis of providing functions that meet the needs of users is essentially icing on the cake. Enhancing communication with users and strengthening the user's desire to use the product is a great significance of boosting the product and user interaction. In addition, it's important to show a designer's subjective initiative to convey more vivid product information to users. In the mean time, it's necessary to embrace a comprehensive understanding of information and do what customers like. As long as you know what the user likes, it's possible to attract him to use. The more comprehensive and in-depth understanding of the target user's information are grasped, the better communication with the users can be reached.

*Project category: 2018 Special Scientific Research Plan (Humanities and Social Sciences Special Project) of Shaanxi Provincial Department of Education

Project name: Research on interactive design of Shaanxi coal logistics platform based on user experience.

Project number: 18JK0725

Project leader: Shen Yan

\section{INTERACTIVE DESIGN PRINCIPLES OF COAL LOGISTICS PLATFORM}

\section{A. The Clarity of Function Buttons and the Vivification of Feedback}

At present, a large part of the truck drivers in China are self-employed and engaged in logistics transportation. However, due to the relatively poor anti-risk ability of individual business, and the unstable supply of goods, low bargaining power and relatively backward means of delivery and other factors, some of drivers' survival situation is worrying. From the quality of life, it is mainly explained in bad habits, irregular life and rest, overloaded work and so on. Because of the influence of the working environment, the working conditions of the coal logistics drivers are worse. Perennial road driving, relatively monotonous work content and huge freight tasks make truck drivers prone to tension and anxiety. Because of the limitations of work, most truck drivers lack the opportunity to communicate with the outside world, resulting in their physical and psychological problems, which seriously affect their physical and mental health.

\section{B. Making Behavioral Input Simplest to Minimize User Workload}

The first is to seek truth and low price. Because of the difficulty of making money, truck drivers want to increase transportation costs and provide them with corresponding preferential policies, such as, the exemption of freight information service fees, the supply of certain insurance services and so on

The second is to pursue simplicity and convenience. Drivers prefer more simple and understandable behavior in the use of products, and generally repel the use of more complex platforms. Meanwhile, the product's function is best to have a certain degree of guidance, easy for drivers themselves to operate. As a result, platforms that are apt to use, learn, and operate tend to be supported by drivers.

The third one is relatively strong mood swings. Trucks have no timetables, so that the transport process at any time may be in a state of shutdown. Besides that, lack of good source of goods can also lead to shutdown, which not only increases their economic losses, but also worsen their psychological impact. 


\section{Consistency Principle}

Consistency principle plays an important role in the interactive design of coal logistics platform. It can help users to use their own experience in new usage scenarios, reduce the memory burden as well as the frequency of misoperation, so that users are allowed to adapt to the operating habits of products faster and more easily accept applications. Most users of coal logistics platforms are elder ones. So, in the design process, it's necessary to pay attention to the consistency of interface vision, function, information architecture, operation and so on to improve the driver's recognition and resolution. Visual design also needs to ensure the consistency principle of color and graphics. And in the mean time, in the design of the interface, the consistency of color, layout and use of elements, etc should be kept. The information of the same system in interface and the information expression of the same function in different interfaces shall be consistent. At the same time, it is necessary to keep the structure and logic of all levels consistent. In addition, users need to maintain consistency when performing user tasks of the same attribute.

\section{VISUAL DESIGN PRINCIPLES OF COAL LOGISTICS PLATFORM MEETING USER NEEDS}

\section{A. Interface Format}

Based on the user's operating habits and visual habits, in the layout design of the interface, most of the important content is placed above and below the mobile phone interface, easy for users to operate. At the same time, the different mobile devices also directly affect the user's experience, for example, if the screen button resolution is lower than 44 pixels, it is easy to cause user misoperation. In order to reduce such situation, the key resolution should be as large as possible than this value. In addition, the mobile application interface is usually top-down in layout. The layout design principles of coal logistics platform interface design can be divided into three aspects. First, the layout of elements is symmetrical. The layout of pictures and words should be distributed in four directions of the interface and the number should be equal. But this layout sometimes appears rigid to some extent. In order to avoid this situation, some adjustment can be made to disrupt the layout of the original elements and create a balanced effect. Secondly, each element should be mutually corresponding. The principle of change emphasizes the echo of the beginning and the end of the layout design. In the beginning, some elements are designed and used in the interface. In the following interface, there should also be the existence of these elements, to achieve the effect of the first and last echo, realizing the interface uniformity. The third one is contrast. In the design of the interface, different graphic elements, graphic symbols and colors are compared and designed, and they are integrated into the design of the interface to form the diversity of the interface, so that the interface gains richness visually and exempt from singleness.

\section{B. Graphic Symbol Design}

Graphic symbols play an indicative role as a medium of interpretation and visual communication, using graphic symbols to stimulate the visual and perceptual level of the user. The communication medium, the object of expression and the meaning of interpretation are the three basic elements of graphic symbols, which fundamentally reflect the whole process of human thinking. Graphical semiotics originated from the linguistics of the past. Structural linguistics, logic, cultural philosophy and aesthetics have become parallel to modern graphic semiotics. It is an emerging discipline that studies symbolic systems.

Combined with the characteristics of the driver user, the square corners of the graphical symbols are reminiscent of calm, composed male features. Therefore, the graphic design of the coal logistics platform is often expressed by the square block surface.

With the continuous development and improvement of the modern Internet, today's graphic symbol research has gradually transformed from the traditional single language symbol to the modern diversified visual image symbol. The diversity of language symbols includes graphics, color, motion and text, while graphic symbol language is widely adopted and continuously developed in modern mobile product design. The reason lies in that graphic symbol language of visual symbols have more propagative and direct visual expression characteristics than other symbols. To design an easily recognizable graphical visual symbol, it is necessary to delve into and summarize the essential relationship between the symbol of the object and the user, and whether the symbol and the user's functional requirements are suitable. In short, when designing the driver terminal of the coal logistics platform, the graphical symbols should be simple and easy to identify. The design of the graphics should be rich in form and needs to be summarized, making it easy for the freight driver to understand and accept the meaning expressed by the graphics, so that it can be closer to the user cognition. At the same time, the design of the graphic needs to be simple and easy to understand, and the simple graphic should be used to express the intention, and the size should be guaranteed within the visual acceptance of the user with clear distinction of the primary and secondary composition.

\section{Color Application}

In mobile application product design, color is an important element for people to make basic judgments and information processing decisions on the object of expression, so as to judge the basic state and specific feeling of things. Most truck drivers are male users, and they have a low perception of color. Survey data show that men's perception of color is generally lower than that of women. Men tend to pursue a more rational cold tone, so cold tone can be used as the main color tone of the product.

The human brain responds faster to color than sounds and symbols. Modern design expresses emotion by stimulating human perception and color is the most direct element to stimulate perception. The human brain can quickly process 
the visual information conveyed by colors. When the human eye perceiving colors, it will reflect potential mental impressions in the brain constantly. Therefore, in visual design, the use of color is very common and important. The color effect and color expression of product design depend on the color composition and expression to a certain extent. At the same time, there are many differences in color, not only about saturation, but also brightness. These differences also have diverse psychological effects on drivers, such as: cold and warm, far and near, hard and soft. Blue belongs to a cool color system in the whole color system, reminding people of the sea and sky, giving people a quiet, wise, calm, profound visual feeling; red reflects a warm tone with enthusiasm and unrestrained meaning; yellow also reflects warmth. In ancient times, yellow was the symbol of imperial power; green gives a feeling of relaxation, calmness and nature; white makes people feel pure, noble; purple is a cold color, conveying an elegant and noble feeling; orange makes people feel happy.

In the design of the mobile interface product, it is necessary to fully consider that the disorganized color matching may cause a very bad user experience, and the user will have an antipathy to the application product due to visual fatigue, resulting in the failure of entire mobile interface product. Therefore, in the design of mobile products, it's obligatory to first achieve the harmony and unification of visual colors. In color matching, it's wise to select the same color system. First, the main color of the product should be determined, and then it's important to select the contrast color of the main one to show the decoration and color complement of each other, playing a role of embellishment and coordination in small area.

At the same time, for hue matching, beyond the unification in the color style, it's better to enhance the color layering of the application interface. Seeking change in unity will make the interface free from boredom. The tone and color of the product should be bright, so that the tincture character can be clearly expressed to satisfy the product users' love for the color of the product. Except that, most of the driver users are males. Studies have shown that men prefer the color combination of cool ones, and the low purity and low-light colors are more suitable for their aesthetic needs. Contrast color refers to the two colors in the color circle that are diagonal. In modern product design, the complementary color visual effect will produce the strongest color contrast effect in color matching. If it is cleverly used, it will make the interface produce the strongest visual impact. The complementary color is also an important matching method of interface composition, which cannot be ignored.

\section{Text Design}

As the most basic recognition symbol of information transmission in human society, the application of characters in mobile interface is a very important part. Text design in the application interface design mainly appears in the main interface, menu bar, navigation bar, icon name, card surface content, details page content interface. Coal logistics drivers are a group of people with a relatively direct mode of thinking. They tend to have a low level of education and are mostly middle-aged men. These people are easily moved by intuitive things. Even giving intuitive and easy-to-understand text in the app interface will impress them. Conversely, complex and variable and descriptive texts will be subject to their psychological exclusion and visual aversion.

In the text design of the cargo driver's interface, the text first requires simplification and easy understanding. In the application interface of the logistics platform, about twothirds of the text is fixed text, which cannot be edited during visual design. Therefore, the text design and semantics fail to allow the expression of driver's favorite style. The basic text size, text color and graphic text are only ways to reflect their tastes and it's important to fully guarantee the text to be readable and easy to recognize. The file size of the graphic text also needs to be reasonably designed to improve the loading speed of the product runtime and save the user's waiting time.

Words as communication symbols, when used in the small visual communication interface of mobile phone, have a higher requirement on the accuracy and intuition of information transmission. For drivers, the accuracy of logistics freight information is quite critical. Besides that, the size, depth and font of the text should be kept consistency. For drivers of coal logistics platform, through the investigation, it is found that the font color should be blue, gray and black, which accords with the male users' preference. In addition, reasonable typesetting design, unification, symmetry, change and other basic composition principles are important design techniques for reasonable combination of words and graphics.

\section{E. Animation and Sound Effects}

Animation means a picture in motion. That is to say, it can show a certain dynamic effect. When designing, it's crucial to define the object of movement. Firstly, the moving objects shall be distinguished from the static ones, and the stationary objects and the different moving objects should be placed in different groups, so as to avoid interference between them. After identifying the objects, it's necessary to do best to make them move. For individual object, its location, speed, movement mode etc. should be identified. For multiple objects, in addition to the information required by individual one, we also need to know the relationship between multiple objects.

There are two kinds of dynamic effects in mobile applications. All the strange dynamic effects we see are the results of combining and multiplying the two. In terms of truck-to-driver user characteristics, animation effects on the platform can be changed in size, shape, transparency, brightness and color. Assembling these ways together will produce a variety of effects. Because the driver has been driving for a long time and the work is boring, sometimes there will be restless mood, so, in the design of effect, the sound should relatively soft to meet the needs of the driver user. 


\section{CONCLUSION}

Whether for product or interface design, determining the target user is the most important thing. The role of user has a great impact on the product, so, designing different Internet products for different target audiences can help to improve the user experience of the product. In the design of coal logistics platform products, the relevant personnel should not only consider the gather of massive information source, but also need to consider the real needs of users and aesthetic experience and other elements, so as to bring users as much as possible a good operating experience. Therefore, it is of great practical significance to explore how to design the application software of coal logistics platform with good user experience.

\section{REFERENCES}

[1] Li Binbin. Design Psychology [M]. China Light Industry Press.2001.

[2] Geerig R, Zimbardo P. Psychology and Life [M]. Wang Lei et al.translates. People's Posts and Telecommunications Press 2003.

[3] Cooper A, Reimann R, Cronin D. About Face 3: The Essence of Interactive Design $[\mathrm{M}]$. Liu Songtao et al. translate. Electronic Industry Press 2008 (18).

[4] Galotti K. Cognitive Psychology [M]. Shaanxi Normal University Press, 2005 (10).

[5] Solomon M. Consumer Behavior [M]. Renmin University of China Press, 2009.

[6] Garrett J J. User Experience Elements [M]. Fan Xiaoyan Translates. Mechanical Industry Press. 2007.

[7] Cagan J, Vogel C. Creative Breakthrough Products [M]. Xin Xiangyang, Pan Long translate. Mechanical Industry Press, 2004.

Norman D. Emotional Design [M]. Fu Qiufang et al. translate. Electronic Industry Press, 2005. 